Published in final edited form as:

Ann Epidemiol. 2008 September ; 18(9): 671-677. doi:10.1016/j.annepidem.2008.06.006.

\title{
HDL-cholesterol and incidence of breast cancer in the ARIC cohort study
}

Anna M. Kucharska-Newton, PhD MPH, Department of Epidemiology, University of North Carolina at Chapel Hill, Chapel Hill, NC

Wayne D. Rosamond, PhD, Department of Epidemiology, University of North Carolina at Chapel Hill, Chapel Hill, NC

Pamela J. Mink, PhD, Department of Epidemiology, Emory University, Atlanta, GA

Anthony J. Alberg, PhD MPH, Hollings Cancer Center, Medical University of South Carolina, Charleston, SC

Eyal Shahar, MD, MPH, and Division of Epidemiology and Biostatistics, Mel and Enid Zuckerman College of Public Health, University of Arizona, Tucson, AZ

Aaron R. Folsom, MD, MPH Department of Epidemiology, University of Minnesota, Minneapolis, MN

\section{Abstract}

PURPOSE-An association of low plasma HDL-cholesterol with risk of breast cancer has been suggested by multiple studies; the evidence, however, is not conclusive. We examined the possible association of low HDL-cholesterol with incidence of breast cancer using data from the Atherosclerosis Risk in Communities Study (ARIC) cohort, a prospective study of a randomly selected sample of women and men from four US communities.

METHODS-Among 7,575 female members of the ARIC cohort, 359 cases of incident breast cancer were ascertained during the follow-up from 1987 through 2000. In analysis adjusted for age, race, body mass index, smoking, and reproductive variables we observed no association of low baseline HDL-cholesterol $(<50 \mathrm{mg} / \mathrm{dL})$ with incident breast cancer in the total sample (HR=1.08(95\% CI $0.84,1.40))$ and a modest association ( $\mathrm{HR}=1.67(95 \%$ CI 1.06, 2.63) among women who were premenopausal at baseline. No association was observed among women who were post-menopausal at baseline. Removal from analysis of the first five years of follow-up did not appreciably change the observed associations.

CONCLUSION—Results of our study suggest that low HDL-cholesterol among pre-menopausal women may be a marker of increased breast cancer risk.

\section{MeSH headings \\ Breast Neoplasms; Cholesterol; HDL; Incidence; Cohort Studies}

Correspondence to: Anna M. Kucharska-Newton.

Publisher's Disclaimer: This is a PDF file of an unedited manuscript that has been accepted for publication. As a service to our customers we are providing this early version of the manuscript. The manuscript will undergo copyediting, typesetting, and review of the resulting proof before it is published in its final citable form. Please note that during the production process errors may be discovered which could affect the content, and all legal disclaimers that apply to the journal pertain.

Disclosures: none 


\section{Background}

Identification of modifiable risk factors for breast cancer is hampered by complexity of the disease and a long lag period between exposure and clinically apparent disease $(1,2)$. Currently recommended interventions aimed at reducing the risk of breast cancer are weight management, exercise, reduction in alcohol intake, and, for post-menopausal women, modifications in hormone replacement therapy regimen. The obesity epidemic has focused attention on the role of obesity in breast cancer risk. Measures of obesity are consistently associated with an increased risk of post-menopausal breast cancer (3). Association of obesity with the risk of pre-menopausal cancer, differ by race, with an inverse association observed among white women and no association among African-American women (3). Possible mechanisms explaining the role of adipose tissue in the etiology of breast cancer involve an increase in estrogen synthesis $(4)$, increase in insulin levels $(4,5)$, and a decrease in adiponectin levels (6). Obesity affects the lipid profile (5,7), and decreases levels of HDL-cholesterol. Recent research suggests that low HDL-cholesterol, a traditional cardiovascular disease risk factor, may be associated with incidence of cancer at various sites (8) and, as such, may constitute a potential marker of elevated cancer risk. Of the four prospective studies examining the association of low HDL-cholesterol with the risk of breast cancer, two have stratified by menopausal status. One of these (9) suggested that the risk of breast cancer is increased among pre-menopausal women with low HDL-cholesterol, while the other reported an association of low HDL-cholesterol with increased risk of post-menopausal breast cancer (10). Studies not stratified by menopausal status reported mixed results $(11,12)$. This variation in reported results may reflect differences in study population, study design, as well as selection of analysis covariates. In addition to these prospective studies, several case-control studies have shown an association of low HDL-cholesterol with increased breast cancer risk $(8,13-16)$.

The following study, aimed at clarifying the existing evidence, is based on the Atherosclerosis Risk in Communities (ARIC) cohort, a longitudinal multi-center, bi-racial study with well defined baseline covariates and a long follow-up period.

\section{Materials and Methods}

This study was approved by the appropriate Institutional Review Boards and all participants provided written consent.

\section{Study description and baseline measurements}

The ARIC cohort consists of 15,792 men and women, 45-64 years of age at baseline (19871989), selected as a probability sample from four US communities located in North Carolina, Mississippi, Minnesota and Maryland (17).

The cohort was examined and risk factor assessed at baseline and at three subsequent triennial visits through 1998. Risk factors for this analysis were measured at baseline unless indicated otherwise.

Blood drawn from an antecubital vein into tubes containing EDTA and plasma obtained by centrifugation at $4{ }^{\circ} \mathrm{C}$ and stored at $-70^{\circ} \mathrm{C}$ until analysis. Plasma HDL-cholesterol levels were measured using the method of Warnick et al (18). HDL-cholesterol was coded as a continuous variable (hazard ratio per one standard deviation), as quartiles of distribution (cutpoints: $<45.2$ $\mathrm{mg} / \mathrm{dL}, \geq 45.2-55.0 \mathrm{mg} / \mathrm{dL}, \geq 55.0-67.4 \mathrm{mg} / \mathrm{dL}, \geq 67.4 \mathrm{mg} / \mathrm{dL}$ ), and as "high" or "low" based on the cardiovascular risk clinical cutpoint $(50 \mathrm{mg} / \mathrm{dL})(19)$. Plasma triglyceride levels were determined enzymatically (20) using the Cobas Bioanalyzer (Roche). Body mass index was calculated as the ratio of weight in kilograms to height in meters_squared.. Waist and hip 
circumferences were measured with the subject standing. Waist-hip ratio was calculated as a ratio of waist circumference at the umbilicus to the circumference measured at the widest part of the hips.(21) . Diabetes status (yes/no) at baseline was based on either one of the following criteria: fasting plasma glucose levels $\geq 126 \mathrm{mg} / \mathrm{dL}$, non-fasting plasma glucose $\geq 200 \mathrm{mg} / \mathrm{dL}$, use of anti-diabetic medication within two weeks of baseline data collection, or self-report of a physician diagnosis of diabetes. hypertension was defined by current use of antihypertensive, or systolic blood pressure $\geq 140 \mathrm{~mm} \mathrm{Hg}$, or diastolic blood pressure $\geq 90 \mathrm{~mm} \mathrm{Hg}$. Level of exercise was based on reported level of sport activity using the Baecke physical activity questionnaire (22). Alcohol consumption was based on self-report of beer, wine and liquor consumption. Participants were classified as current, former and never smokers at baseline and cigarette years of smoking were defined as average number of cigarettes smoked per day times number of years smoked.

\section{Reproductive variables}

Menopausal status was defined as: pre-menopause, peri-menopause, natural post-menopause, and surgical post-menopause (23). We grouped women who were pre-menopausal $(n=1,494)$ and peri-menopausal $(\mathrm{n}=723)$ at baseline into one category which we labeled "pre-menopause" and those with both natural and surgical menopause at baseline into a category labeled "postmenopause". In order to validate our placement of peri- menopausal women together in a group with pre-menopausal women we repeated our analyses excluding those cohort members who were peri-menopausal at baseline.

Information concerning hormone replacement therapy use, number of live births, age at menarche, and age at menopause was obtained at baseline. Age at first birth and history of bilateral breast cancer in a first degree relative were obtained by a supplemental questionnaire collected during telephone follow-up interviews conducted in 1994. Of the 8,710 female members of the ARIC cohort 8,140 (93\%) responded to this questionnaire.

\section{Incident breast cancer cases}

Incident breast cancer was ascertained from 1987 through 2000 based on linkages to the following cancer registries: the Minnesota Cancer Surveillance System, the North Carolina Cancer Registry, the Washington County (Maryland) Cancer Registry, the (statewide) Maryland Cancer Registry, and the Mississippi Central Cancer Registry (from 1995). Breast cancer cases prior to registry initiation (Jackson, Mississippi from 1987 until 1995; Minnesota from 1987 to 1988) and other possible missed cases were identified through review of all ICD codes of hospitalizations reported by ARIC study and medical record retrieval. (24).

\section{Statistical analysis}

Of 8,710 female ARIC study members at baseline, we excluded those with races other than white or black $(n=25)$; those who fasted less than 8 hours before venipuncture $(n=262)$, or had missing data on fasting status $(n=44)$; missing HDL-cholesterol status $(n=164)$; missing cigarette smoking status $(n=9)$; those with prevalent cancer $(n=577)$; or those missing information concerning breast cancer incidence $(n=117)$. The final cohort for this analysis included 7,575 women. In analyses stratified by menopausal status, women with unknown menopausal status $(n=913)$, as well as women with primary amenorrhea $(n=4)$ were further excluded, yielding 6,658 as the final number of study participants

Baseline characteristics were summarized as means and proportions of selected variables. Pairwise t-tests and Pearson's $\chi^{2}$ coefficients were used to compare means and proportions of baseline characteristics among the exposed and unexposed groups. 
Associations between baseline HDL-cholesterol and breast cancer incidence were initially examined using Kaplan-Meier survival estimates. Cox proportional hazard regression of the association of low HDL-cholesterol $(<50 \mathrm{mg} / \mathrm{dL})$ and breast cancer incidence was performed with evaluation of the following baseline variables for inclusion: age (continuous), race (black, white), body mass index (continuous), waist-hip ratio diabetes (yes, no), log triglycerides, smoking status (current, former, never), alcohol intake (grams per week), sports score (ordinal), education (less than high school versus high school or greater), age at menarche (continuous), age at menopause (continuous), and number of live births (continuous). Variables were considered fro inclusion into the regression model on the basis of existing literature and retained according to a $10 \%$ change-in-estimate criterion (25). Adherence to proportional hazard assumptions was determined for each covariate and for the overall regression models on the basis of smoothed plots of Schoenfeld residuals and log-negative log plots (26) performed before and after stratifying on menopausal status. We evaluated the final fit of the models by plotting partial Cox-Snell residuals. Tests of the linear trend for the association between HDL-cholesterol and breast cancer incidence were performed using Cox regression models with HDL-cholesterol coded as a continuous variable or as an ordinal variable indicating HDL-cholesterol quartiles.

Interactions between HDL-cholesterol and selected covariates were evaluated using likelihood ratio tests comparing models with and without multiplicative interaction terms, and by comparing expected and observed incidence rates assuming multiplicative effects.

All analyses were conducted using STATA statistical software package, version 8.0 (STATA Corporation, College Station, TX).

\section{Results}

There were 359 incident breast cancer events identified in during follow-up from 1987 through 2000. Low baseline HDL-cholesterol (less than $50 \mathrm{mg} / \mathrm{dL}$ ) was associated with greater current smoking, higher mean triglyceride levels, higher average BMI and waist-hip-ratio, higher prevalence of diabetes, and lower use of hormone replacement therapy (Table 1). The ratio of post-menopausal women to pre-menopausal women was 2.11 for cases (224/106) and 2.00 for non-cases $(4,217 / 2,111)$. Eight percent of the cases and 12.3 percent of the non-cases were missing information on menopausal status.

Sizeable differences between pre-menopausal and post-menopausal women (Table 2) existed for age, race, triglyceride levels, hormone replacement therapy, diabetes, and education.

We performed a formal evaluation of potential effect modification of the association of low baseline HDL-cholesterol and incident breast cancer for the following covariates: menopausal status, smoking (ever versus never), body mass index (using the $25 \mathrm{~kg} / \mathrm{m}^{2}$ cutpoint), hormone use (ever versus never), diabetes status, and race. We found no evidence of effect modification, except a very modest supra-multiplicative effect of menopausal status $(\mathrm{p}=0.04)$. Specifically, an association between low HDL-cholesterol and incidence of breast cancer was only observed among women who were pre-menopausal at baseline (Figure 1). This observation agrees with results of Cox proportional hazard regression analysis which suggests a modest association of low HDL-cholesterol with increased incidence of breast cancer among women who were premenopausal at baseline (HR=1.67 (95\% CI 1.06, 2.63) (Table 3).

Analysis of adjusted hazard rate ratios of breast cancer incidence in quartiles of HDLcholesterol levels (Table 3 ) confirmed the lack of an association in women who were postmenopausal at baseline and suggested a possible gradient of association in the first three quartiles of HDL-cholesterol in women who were pre-menopausal at baseline. No was 
observed in either strata of baseline menopausal status when HDL-cholesterol was coded as a continuous variable.

We addressed the possibility of reverse-causality in the association of low HDL-cholesterol and breast cancer incidence by removing from our analysis the first five years of follow-up (Table 3). The adjusted hazard ratio for low versus high HDL-cholesterol was 1.34 (95\% CI $0.98,1.82)$ for the total sample, $2.25(95 \% \mathrm{CI} 1.31,3.86)$ for the pre-menopausal women and 1.07 (95\% CI $0.71,1.63)$ for the post-menopausal women.

\section{Sensitivity analyses}

Adjustment of analysis for age at first pregnancy slightly strengthened the observed hazard rate ratios in pre-menopausal women and did not substantially alter the association among postmenopausal women (hazard rate ratio in fully adjusted model: pre-menopausal women 1.76 (95\% CI 1.09, 2.84), post-menopausal women: 0.97 (95\% CI 0.67, 1.40)). Further adjustment for the waist-hip ratio, triglycerides, family history of bilateral breast cancer diagnosis, and use of cholesterol lowering medications did not appreciably alter the results. We did not observe any significant differences in the hazard ratios calculated when women who were perimenopausal at baseline ( $\mathrm{n}=723$ ) were excluded from the analysis (HR 1.99 (95\% CI 1.13, 3.52).

In order to eliminate the potential effect of exogenous estrogens, we repeated our analyses in a subset of the study sample - women who were pre-menopausal at baseline and who had never received hormone replacement therapy. The resulting hazard ratio for low HDL-cholesterol, 2.15 (95\% CI 1.31, 3.52) was greater than that observed for the full cohort of pre-menopausal women, although the difference between the two estimates was not statistically significant.

In this cohort of 7,575 women, 913 were missing information concerning menopausal status (886 women with unknown ovarian status, 27 women with missing data on menopausal status). A repeat analysis of the association of low HDL-cholesterol with incidence of breast cancer based on categories of age rather than menopausal status, with 50 years as the age cutpoint, performed to avoid potential misclassification bias due to the amount of missing data, yielded results very similar to those obtained using menopausal status data.

There were 18 cases of breast cancer identified in the Jackson, Mississippi, center prior to establishment of the Mississippi Central Cancer Registry and five cases of incident breast cancer recorded prior to establishment of the Minnesota Cancer Surveillance System. Removing those cases from analyses did not appreciably change the effect estimates.

\section{Discussion}

This prospective cohort study observed a modest association of low-HDL cholesterol with increased incidence of breast cancer among women who were pre-menopausal at baseline $(\mathrm{HR}=1.67,(95 \%$ CI: 1.06, 2.63).

Of the previous prospective studies Moorman et al. (9) reported a modest association of low HDL-cholesterol with increased risk of breast cancer among pre-menopausal women, while Furberg et al (10) suggested of an association of low-HDL cholesterol and increased breast cancer incidence only among post-menopausal women The analysis by Furberg et al. involved a comparison of relative risks of the disease in quartiles of HDL-cholesterol level. The relatively small point estimates and small number of cases per quartile do not exclude the possibility of a modest association among pre-menopausal women as well. The authors of that study observed an association only among post-menopausal women with body mass index levels greater than $25 \mathrm{~kg} / \mathrm{m}^{2}$. It is possible that, despite adjustment for body mass index, this observation reflects a residual effect of obesity not captured by the BMI classification, 
especially abdominal obesity (27). Our results appear consistent with data from the study by Moorman et al (9). The fact that we also observed the association of low HDL-cholesterol and the risk of breast cancer in a sample in which the first five years of follow-up have been removed from analysis would suggest independence from the effect of preclinical disease on levels of HDL-cholesterol.

Our observation of a modest association of low HDL-cholesterol with increased incidence of breast cancer among women who were pre- and peri-menopausal at baseline, but not among post-menopausal women, can perhaps be explained in the context of the hormonal regulation of breast cancer. High endogenous estrogen levels are one of the strongest risk factors for breast cancer(1). High estrogen levels, observed during pre-menopause, are inversely associated with low HDL-cholesterol (28). Menopause leads to a decrease in levels of endogenous estrogen, thereby reducing the potential effect of HDL-cholesterol as a marker of endogenous estrogen activity.

HDL-cholesterol levels have been found to be inversely associated with levels of insulin-like growth factor-I (IGF-I) (29-31). Existing studies suggest the presence of an association between IGF-I among pre-menopausal women and subsequent incidence of breast cancer during post-menopausal years (32). High premenopausal levels of IGF-I are also associated with increased breast density during post-menopausal years (33). Increased mammographic breast density has been found to be independently associated with an increased risk of breast cancer (34). Our results, suggesting a modest association of low HDL-cholesterol with breast cancer incidence among women who were pre-menopausal during baseline data collection, would support those observations.

The $50 \mathrm{mg} / \mathrm{dL}$ cutpoint for the dichotomous HDL-cholesterol variable was chosen on the basis of its common use in cardiovascular disease risk stratification. To our knowledge, this cutpoint does not have a biological significance associated with the risk of breast cancer. Analysis of the association of HDL-cholesterol as a continuous variable, as quartiles of HDL-cholesterol distribution, or through spline regression analysis did not confirm the results we obtained with the dichotomous HDL-cholesterol classification. Those inconsistencies may have arisen as a result of a small number of incident cases of breast cancer among women who were premenopausal at baseline $(\mathrm{n}=106)$.

The strengths of this study include its prospective design and the biracial aspect of a cohort drawing from four diverse communities in the United States. HDL-cholesterol measurement variability in the ARIC study has been estimated to be very small (5\%) (35). Limitations of this study reflect lack of information concerning type and stage of breast cancer, relatively small number of cases of breast cancer among women who were pre-menopausal at baseline data collection, and lack of information concerning menopausal status at time of breast cancer diagnosis. There is no information in the ARIC study concerning screening for breast cancer. It is possible that detection of breast cancer was different for pre-as compared to postmenopausal women. It is difficult however to predict the effect any such differential misclassification would have had on the association of HDL-cholesterol with breast cancer.

In conclusion, we have observed a modest association of low pre-menopausal HDL-cholesterol with an increased risk of breast cancer. This association was independent of age, age at menarche, number of live births, race, body mass index, and smoking status, suggesting that metabolic factors associated with lipid metabolism and transport, may have a role in cancer development. 


\section{Abbreviations}

ARIC cohort, Atherosclerosis Risk in Communities cohort; HDL-cholesterol, high density lipoprotein cholesterol; HR, hazard ratio; 95\% CI, 95\% confidence interval.

\section{Acknowledgements}

The Atherosclerosis Risk in Communities Study is carried out as a collaborative study supported by National Heart, Lung, and Blood Institute contracts N01-HC-55015, N01-HC-55016, N01-HC-55018, N01-HC-55019, N01HC-55020, N01-HC-55021, and N01-HC-55022.

The authors thank the staff and participants of the ARIC study for their important contributions.

\section{References}

1. Hankinson, SE.; Hunter, D. Breast Cancer. In: Adami, H-O.; Hunter, D.; Trichopoulos, D., editors. Textbook of Cancer Epidemiology. New York: 2002. p. 301-339.

2. Kelsey JL, Gammon MD. The epidemiology of breast cancer. CA Cancer J Clin. 19911991 May 1;41 (3):146-165.

3. Rose DP, Haffner SM, Baillargeon J. Adiposity, the Metabolic Syndrome, and Breast Cancer in African-American and White American Women. Endocr Rev. 2007Rapid Electronic Publication

4. Calle EE, Kaaks R. Overweight, obesity and cancer: epidemiological evidence and proposed mechanisms. Nat Rev Cancer 2004;4(8):579-591. [PubMed: 15286738]

5. Kaaks R. Nutrition, hormones, and breast cancer: is insulin the missing link? Cancer Causes Control 1996 Nov;7(6):605-625. [PubMed: 8932921]

6. Miyoshi Y, Funahashi T, Kihara S, Taguchi T, Tamaki Y, Matsuzawa Y, et al. Association of Serum Adiponectin Levels with Breast Cancer Risk. Clin Cancer Res. 20032003 November 15;9(15):5699_ 5704.

7. Krause MP, Hallage T, Gama MPR, Sasaki JE, Miculis CP, Buzzachera CF, et al. Association between lipid profile and adiposity in women over age 60. Arquivos Brasileiros de Cardiologia 2007;89:163169.

8. Fiorenza AMBA, Sommariva D. Serum lipoprotein profile in patients with cancer. A comparison with non-cancer subjects. Int J Clin Lab Res 2000;30:141-145. [PubMed: 11196072]

9. Moorman P, Hulka B, Hiatt R, Krieger N, Newman B, Vogelman J, et al. Association between highdensity lipoprotein cholesterol and breast cancer varies by menopausal status. Cancer Epidemiol Biomarkers Prev. 19981998 June 1;7(6):483-488.

10. Furberg A-S, Veierod MB, Wilsgaard T, Bernstein L, Thune I. Serum High-Density Lipoprotein Cholesterol, Metabolic Profile, and Breast Cancer Risk. J Natl Cancer Inst. 20042004 August 4;96 (15):1152-1160.

11. Gaard M, Tretli S, Urdaul P. Risk of Breast Cancer in Relation to Blood Lipids: A Prospective Study of 31, 209 Norwegian Women. Cancer Causes and Control 1994;5(6):501-509. [PubMed: 7827236]

12. Hoyer AP, Engholm G. Serum lipids and breast cancer risk: a cohort study of 5,207 Danish women. Cancer Causes and Control 1992;3:403-408. [PubMed: 1525320]

13. Kumar K, Sachdanandam P, R A. Studies on the changes in plasma lipids and lipoproteins in patients with benign and malignant breast cancer. Biochemistry International 1991;23:581-589. [PubMed: 1877995]

14. Kokoglu E, Karaarslan I, Karaarslan HM, H B. Alterations of serum lipids and lipoproteins in breast cancer. Cancer Letters 1994;82:175-178. [PubMed: 8050088]

15. Dilman VM, Berstein LM, Ostroumova MN, Tsyrlina YV, AG G. Peculiarities of hyperlipidaemia in tumour patients. British Journal of Cancer 1981;43:637-643. [PubMed: 7248149]

16. Ferraroni M, Gerber M, Decarli A, Richardson S, Marubini E, De Paulet P, et al. HDL-Cholesterol and Breast Cancer: A Joint Study in Northern Italy and Southern France 10.1093/ije/22.5.772. Int J Epidemiol. 19931993 October 1;22(5):772-780.

17. The ARIC Investigators. The Atherosclerosis Risk in Communities (ARIC) study: design and objectives. Am J Epidemiol 1989;129:687-702. [PubMed: 2646917] 
18. Warnick GR, Mayfield C, Benderson J, Chen JS, Albers JJ. HDL cholesterol quantitation by phosphotungstate- $\mathrm{Mg}^{2+}$ and by dextran sulfate- $\mathrm{Mn}^{2+}$-polyethylene glycol precipitation, both with enzymatic cholesterol assay compared with lipid research method. Am J Clin Pathol 1982;78:718723. [PubMed: 6182791]

19. American Heart Association. Guidelines C. [cited]; Available from http://www.americanheart.org/presenter.jhtml?identifier $=4500$

20. Nagele U, Hagele EO, Sauer G, Wiedemann E, Lehmann P, Wahlefeld AW, et al. Reagent for the enzymatic determination of serum total triglycerides with improved lipolytic efficiency. J Clin Chem Clin Biochem 1984 Feb;22(2):165-174. [PubMed: 6716056]

21. Ferrario M, Carpenter MA, Chambless LE. Reliability of body fat distribution measurements. The ARIC Study baseline cohort results. Atherosclerosis Risk in Communities Study. Int J Obes Relat Metab Disord 1995 Jul;19(7):449-457. [PubMed: 8520633]

22. Baecke JAH, Burema J, Frijters JER. A short questionnaire for the measurement of habitual physical activity in epidemiological studies. American Journal of Clinical Nutrition 1982;36:936-942. [PubMed: 7137077]

23. Luoto R, Sharrett AR, Schreiner P, Sorlie PD, Arnett D, Ephross S. Blood pressure and menopausal status transition: the Atherosclerosis Risk in Communities study (!987-95). Journal of Hypertension 2000;18:27-33. [PubMed: 10678540]

24. Mink PJ, Shahar E, Rosamond WD, Alberg AJ, Folsom AR. Serum Insulin and Glucose Levels and Breast Cancer Incidence: The Atherosclerosis Risk in Communities Study. Am J Epidemiol 2002;156 (4):349-352. [PubMed: 12181105]

25. Mickey RM, Greenland S. The Impact of Confounder Selection Criteria on Effect Estimation. Am J Epidemiol. 19891989 January 1;129(1):125-137.

26. Hess KR. Graphical methods for assessing violations of the proportional hazards assumption in Cox regression. Stat Med 1995;14:1707-1723. [PubMed: 7481205]

27. Carmichael AR. Obesity and prognosis of breast cancer. Obesity Reviews 2006;7(4):333-340. [PubMed: 17038127]

28. Furberg A-S, Jasienska G, Bjurstam N, Torjesen PA, Emaus A, Lipson SF, et al. Metabolic and Hormonal Profiles: HDL Cholesterol as a Plausible Biomarker of Breast Cancer Risk. The Norwegian EBBA Study. Cancer Epidemiol Biomarkers Prev. 20052005 January 1;14(1):33-40.

29. Kawachi, S-i; Takeda, N.; Sasaki, A.; Kokubo, Y.; Takami, K.; Sarui, H., et al. Circulating InsulinLike Growth Factor-1 and Insulin-Like Growth Factor Binding Protein-3 Are Associated With Early Carotid Atherosclerosis 10.1161/01.ATV.0000154486.03017.35. Arterioscler Thromb Vasc Biol. 20052005 March 1;25(3):617-621.

30. Schernhammer ES, Holly JM, Pollak MN, Hankinson SE. Circulating Levels of Insulin-like Growth Factors, their Binding Proteins, and Breast Cancer Risk. Cancer Epidemiol Biomarkers Prev. 2005 2005 March 1;14(3):699-704.

31. Colao A, Di Somma C, Spiezia S, Rota F, Pivonello R, Savastano S, et al. The Natural History of Partial Growth Hormone Deficiency in Adults: A Prospective Study on the Cardiovascular Risk and Atherosclerosis. J Clin Endocrinol Metab 2006;91(6):2191-2200. [PubMed: 16537686]

32. Rollison DE, Newschaffer CJ, Tao Y, Pollak M, J HK. Premenopausal levels of circulating insulinlike growth factor I and the risk of postmenopausal breast cancer. International Journal of Cancer 2006;118(5):1279-1284.

33. Verheus M, Peeters PHM, Kaaks R, van Noord PAH, Grobbee DE, van Gils CH. Premenopausal Insulin-Like Growth Factor-I Serum Levels and Changes in Breast Density over Menopause. Cancer Epidemiol Biomarkers Prev 2007;16(3):451-457. [PubMed: 17372240]

34. Boyd NF, Guo H, Martin LJ, Sun L, Stone J, Fishell E, et al. Mammographic Density and the Risk and Detection of Breast Cancer. N Engl J Med 2007;356(3):227-236. [PubMed: 17229950]

35. Chambless LE, McMahon RP, Brown SA, Patsch W, Heiss G, Shen Y-L. Short-term Intraindividual Variability in Lipoprotein Measurements: The Atherosclerosis Risk in Communities (ARIC) Study. Am J Epidemiol. 19921992 November 1;136(9):1069-1081.

36. Hoyer AP, G E. Serum lipids and breast cancer risk: a cohort study of 5,207 Danish women. Cancer Causes and Control 1992;3:403-408. [PubMed: 1525320] 
Table 1

Selected baseline characteristics (mean (standard deviation), or percent) of the ARIC study cohort in relation to baseline exposure status

\begin{tabular}{|c|c|c|c|}
\hline \multirow[t]{2}{*}{ Characteristic } & \multicolumn{3}{|c|}{ Exposure status } \\
\hline & Total sample & $\begin{array}{c}\text { Exposed }(\mathbf{n}=\mathbf{2 , 6 8 2}) \\
(\text { HDL-cholesterol<50 mg/dL) }\end{array}$ & $\begin{array}{c}\text { Unexposed }(\mathrm{n}=\mathbf{4 , 8 9 3}) \\
(\text { HDL-cholesterol } \geq \mathbf{5 0} \mathrm{mg} / \mathrm{dL})\end{array}$ \\
\hline Age & $53.7(5.7)$ & $53.8(5.8)$ & $53.7(5.7)$ \\
\hline Race, \% White & 71.0 & 71.9 & 70.6 \\
\hline \multicolumn{4}{|l|}{ Smoking status: } \\
\hline Current & 24.5 & 31.9 & 20.4 \\
\hline Former & 22.3 & 18.5 & 24.4 \\
\hline Never & 53.2 & 49.6 & 55.2 \\
\hline HDL-cholesterol mg/dL & $57.7(17.1)$ & $41.2(6.2)$ & $66.7(14.2)$ \\
\hline High triglycerides $(\geq 150 \mathrm{mg} / \mathrm{dL})(\%)$ & 22.8 & 42.8 & 11.9 \\
\hline $\begin{array}{l}\text { Medication primarily lowering cholesterol } \\
(\%)\end{array}$ & 2.7 & 4.6 & 2.4 \\
\hline All cholesterol lowering medication (\%) & 27.0 & 25.3 & 38.3 \\
\hline Triglycerides (mg/dL) & $122.1(78.6)$ & $162.1(103.2)$ & $100.2(48.6)$ \\
\hline Body mass index & $27.9(6.1)$ & $29.8(6.2)$ & $26.8(5.8)$ \\
\hline Waist hip ratio & $0.90(0.08)$ & $0.93(0.08)$ & $0.88(0.08)$ \\
\hline Diabetes, $\%$ & 10.5 & 18 & 6.4 \\
\hline Education, (\% less than high school) & 23.2 & 27.5 & 20.8 \\
\hline \multicolumn{4}{|l|}{ Menopausal status } \\
\hline Pre- or peri- $(\%)$ & 29.3 & 27.4 & 30.3 \\
\hline Post- (\%) & 56.0 & 59.0 & 58.4 \\
\hline Unknown $(\%)$ & 12.1 & 13.5 & 11.2 \\
\hline Average age at menarche & $12.9(1.8)$ & $12.9(1.7)$ & $12.9(1.8)$ \\
\hline Average age at menopause & $35.1(19.3)$ & $35.3(19.0)$ & $34.9(19.5)$ \\
\hline Average number of live births & $3.3(2.0)$ & $3.2(2.0)$ & $3.4(2.1)$ \\
\hline \multicolumn{4}{|l|}{ Use of hormone replacement therapy } \\
\hline Current Estrogen $(\%)$ & 13.3 & 7.8 & 16.3 \\
\hline Current Estrogen+Progesterone (\%) & 5.5 & 2.9 & 7.0 \\
\hline Never $(\%)$ & 63.9 & 71.2 & 59.9 \\
\hline Former $(\%)$ & 13.9 & 14.9 & 13.4 \\
\hline
\end{tabular}




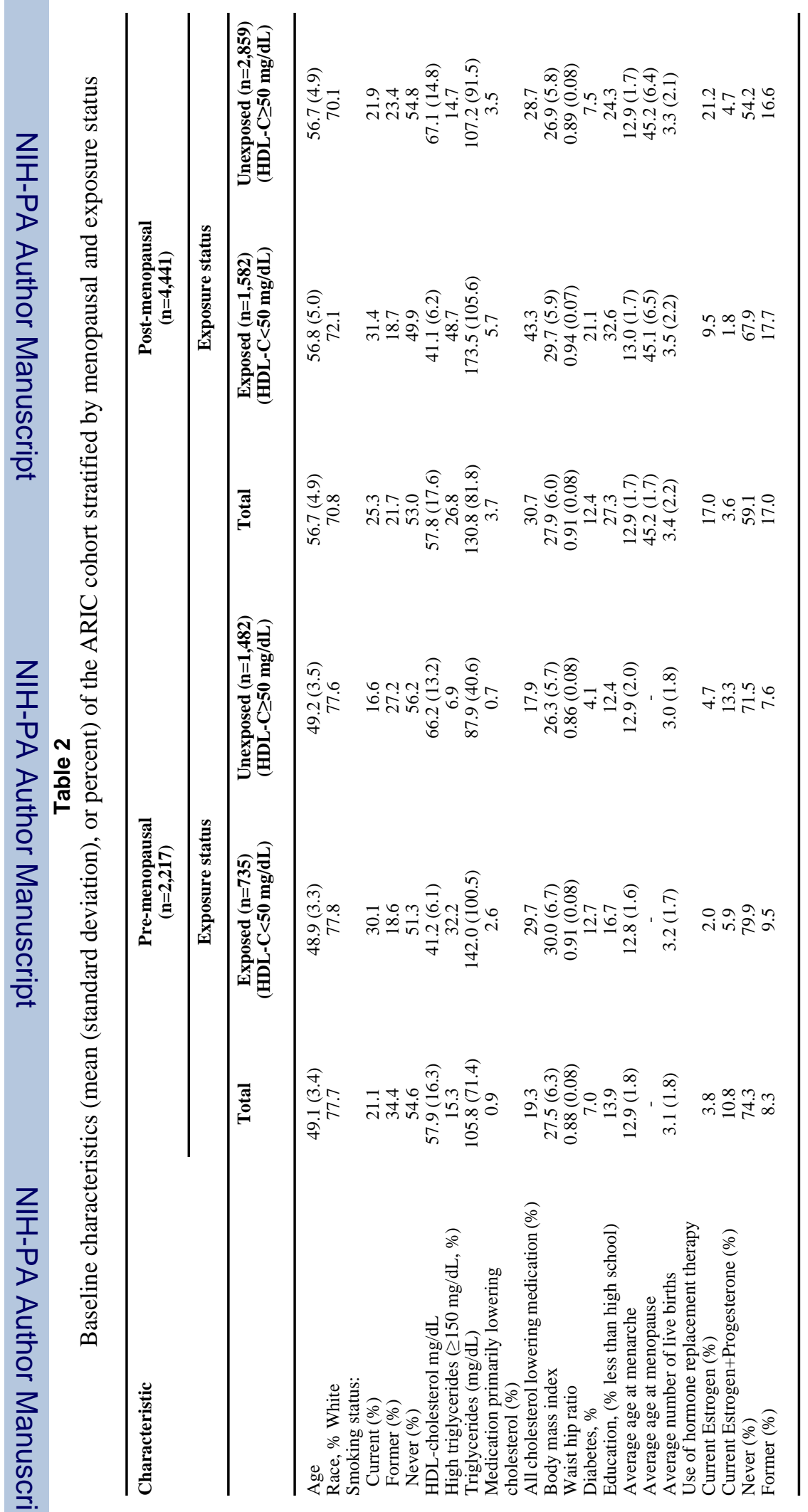

Ann Epidemiol. Author manuscript; available in PMC 2009 September 1. 
Kucharska-Newton et al.

Page 11

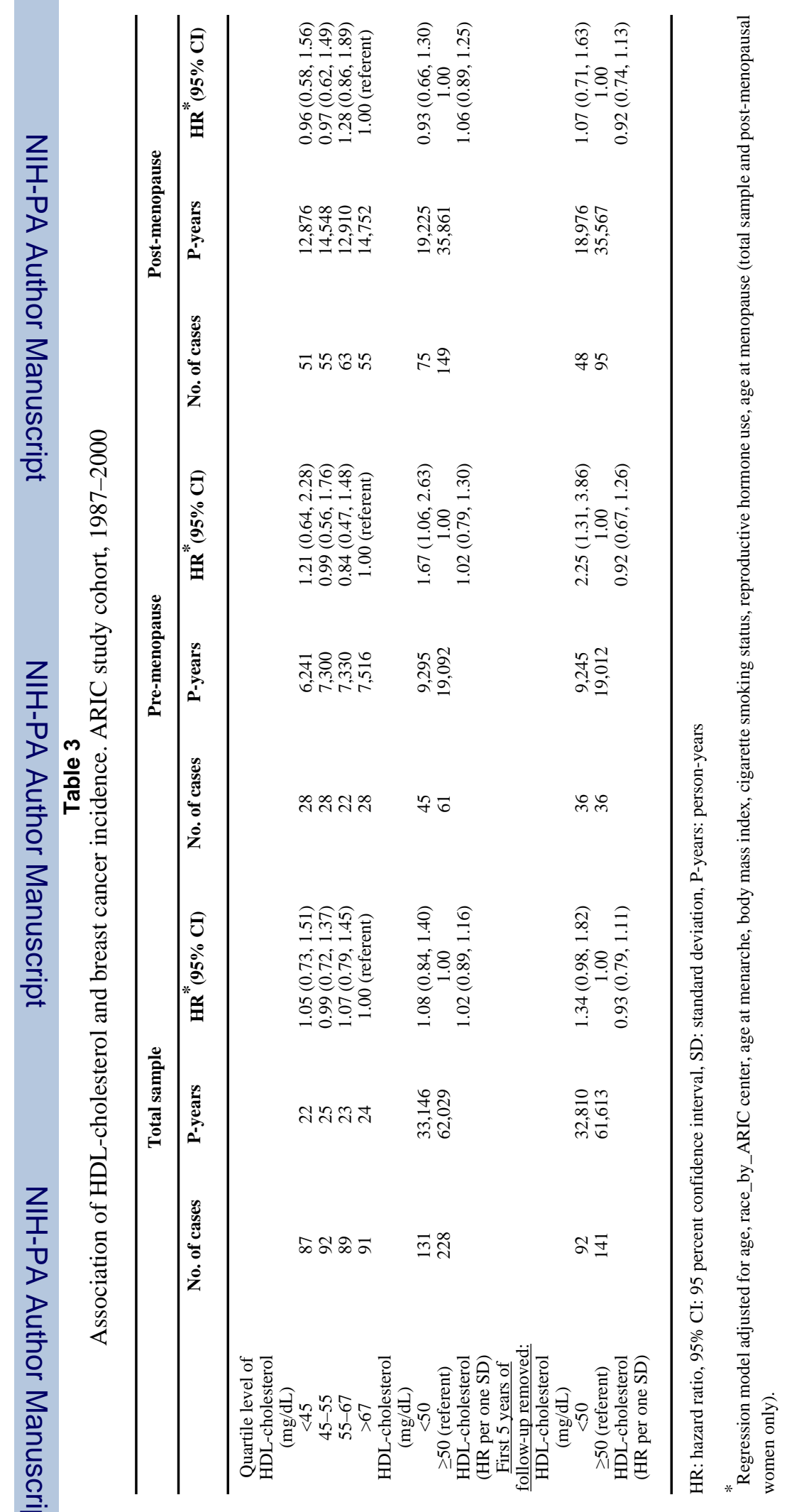

Ann Epidemiol. Author manuscript; available in PMC 2009 September 1. 